\title{
The PDGFC CUB Domain Enhances Survival in PDGFC Mutant Mice
}

\author{
Brian J. Hayes ${ }^{1}$, Kimberly J. Riehle ${ }^{1,2}$, Debra G. Gilbertson ${ }^{3}$, Wendy R. Curtis ${ }^{3}$, Edward J. Kelly ${ }^{4}$, \\ Piper M. Treuting ${ }^{5}$ and Jean S. Campbell ${ }^{1 *}$ \\ ${ }^{1}$ Department of Pathology, University of Washington, Seattle, WA 98195, USA \\ ${ }^{2}$ Department of Surgery, University of Washington, Seattle, WA 98195, USA \\ ${ }^{3} Z$ Zymogenetics Inc, a Bristol-Myers Squibb Company, Seattle, WA 98102, USA \\ ${ }^{4}$ Department of Pharmaceutics, University of Washington, Seattle, WA 98195, USA \\ ${ }^{5}$ Department of Comparative Medicine, University of Washington, Seattle, WA 98195, USA
}

Received: May 01, 2015; Accepted: August 27, 2015; Published: September 14, 2015

*Corresponding author: Jean S. Campbell, Executive Director of Research and Development, Oncosec Medical, 434 N. 34th St, Suite 100; Seattle, WA 98103, USA, Tel: +(206) 221-5422; E-mail: jcampbell@oncosec.com

\begin{abstract}
Absract
Platelet-derived growth factor (PDGF) signaling pathways are necessary for normal development. Here we report a homozygous $P d g f-c$ mutant mouse that is viable. In this mouse, alternative splicing gives rise to a truncated transcript containing the entire coding region of the complement components $\mathrm{C} 1 \mathrm{r} / \mathrm{C} 1 \mathrm{~s}$ sea urchin EGF bone morphogenetic protein 1 (CUB) domain of PDGFC but lacking the majority of the Growth Factor Domain (GFD). A mutant protein is translated from the truncated sequence in vitro. However, the viability of our homozygous mutant Pdgf-c mice depends on the presence of both Pdgfra alleles.
\end{abstract}

Keywords: PDGFC; Cleavage, Growth factor; Knockout

\section{Introduction}

The platelet-derived growth factor (PDGF) signal transduction pathway has important functions in development. Mice lacking PDGF receptor (PDGFR) $-\alpha$ or $-\beta$ are embryonic lethal $[1,2]$ and deletion of Pdgf- $a,-b$, or $-c$ results in perinatal lethality in homozygous Knockout (KO) animals, though live births are seen in Pdgf- $a$ and Pdgf- $c$ KO mice [3-5]. In vitro studies demonstrate that PDGF-AA, -BB, and -CC induce Pdgfr $\alpha$ dimerization, PDGF-BB, and PDGF-DD induce dimerization of $P d g f r \beta$, and $-\mathrm{AB},-\mathrm{BB}$, and -CC induce $\alpha \beta$-receptor dimerization [6]. As predicted by in vitro binding studies, $P d g f-b$ KO mice have a phenotype similar to Pdgf- $b$ KO mice, with defects in kidney and hematologic development $[2,4]$. Mice lacking PDGFA have a defect in lung development [3], and interestingly do not phenocopy deletion of Pdgfr $\alpha$, which causes abnormalities in skeletal development and neural crest migration [1,7]. On the other hand, Pdgf-c KO animals, referred to as $P d g f c^{\text {tminagy }}$, have a defect in palate formation, resulting from abnormal neural crest cell migration and proliferation [5]. Ding, et al. [5], further reported that Pdgf- $a, P d g f-c$ double KO mice have a phenotype similar to Pdgfr $\alpha$ KO mice, suggesting that PDGF-C is a major activator of $P d g f r \alpha$ signal transduction in the context of development.
PDGF-AA, PDGF-BB, and PDGF-AB are secreted as active dimers [8], while PDGF-CC and PDGF-DD are secreted as inactive homodimers requiring extracellular cleavage of an $\mathrm{N}$-terminal complement components $\underline{\mathrm{C}} 1 \mathrm{r} / \mathrm{C} 1 \mathrm{~s}$ sea urchin EGF bone morphogenic protein 1 (CUB) domain to allow receptor binding (Figure 1a)[9-12]. All PDGFs share a common growth factor domain (GFD), which is 110 amino acids long and contains 8 conserved cysteines that facilitate intra- and intermolecular disulfide bonds [13]. PDGF signal transduction is mediated through the GFD, and mutations of these cysteines and loss of their disulfide bonds cause a lack of PDGFR signal transduction $[14,15]$. Furthermore, mutation of sequences in loops I, II, or III of the GFD, the domain that physically interacts with PDGFRs, decreases the GFD's ability to bind PDGFRs [16-18]. Effective signal transduction by $P d g f-c$ thus should require the conserved cysteines and loops I-III of the GFD, as well as cleavage of the CUB domain. We hypothesized that deletion of the majority of the GFD would thus eliminate receptor binding and downstream signal transduction. Contrary to our hypothesis, we describe a homozygous Pdgf-c mutant mouse wherein most of the GFD is indeed deleted, but the expression of the CUB domain in these mice is sufficient for viability. Viability, however, depends on genetic background, as has been described for other Pdgfr $\alpha$ mutations $[19,20]$.

\section{Methods}

\section{Mouse Models}

The Pdgf-c locus was isolated from a 129S5/SvEvBrd genomic BAC library. The targeting construct replaced a $5 \mathrm{~kb}$ genomic region on chromosome 3 with an IRES LacZ MC1 Neo cassette. The homologous arms consisted of a $3 \mathrm{~kb} 5^{\prime}$ fragment and a $2.1 \mathrm{~kb}$ 3' fragment. Lex-1 129S5/SvEvBrd ES cells were transfected with the targeting construct and injected into $\mathrm{C} 57 \mathrm{BL} / 6$ blastocysts to generate chimeras. Chimeras were bred to albino C57BL/6J-Tyr ${ }^{c-2 J}$ mice (The Jackson Laboratory) to generate F1 progeny. F1 hybrids were crossed to generate initial Mendelian ratios (Table 1). Mice 
a PDGF-C

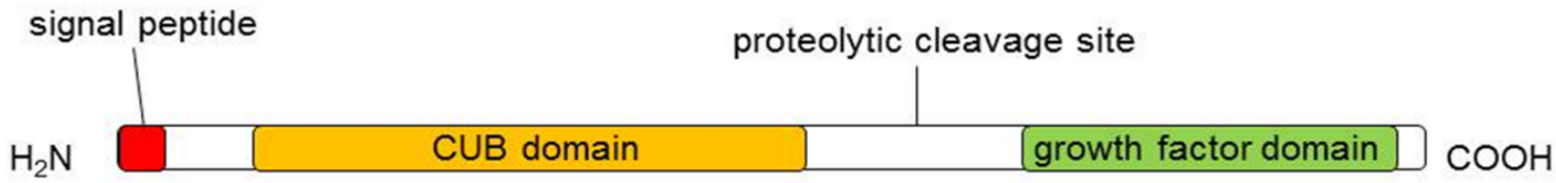

b Pdgfc ${ }^{\text {tm1Nagy }}$ - perinatal lethal

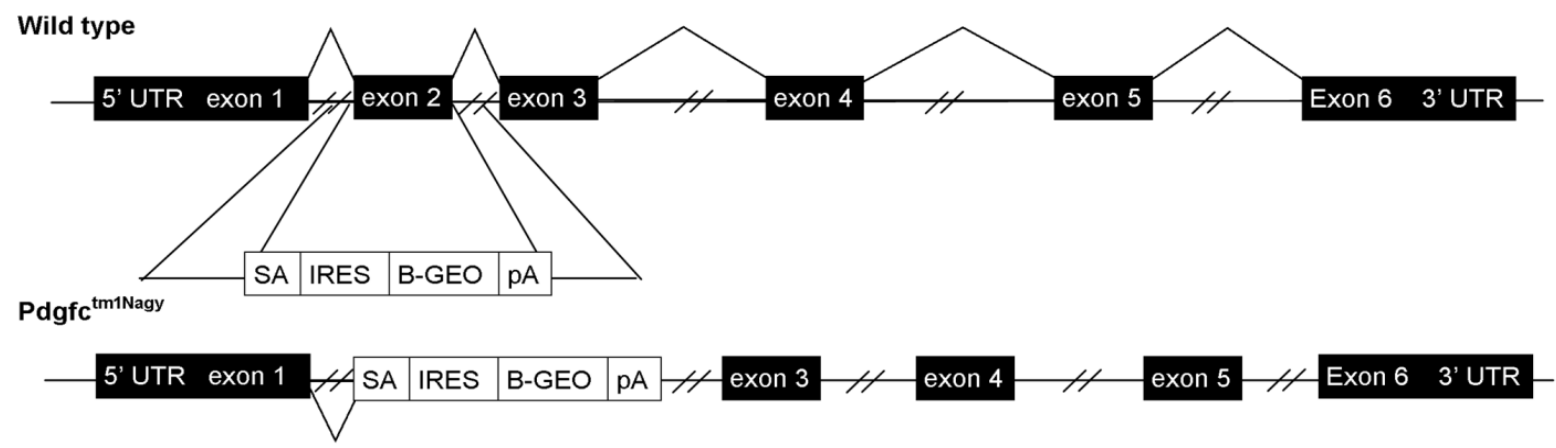

C Pdgfc ${ }^{\text {tm1Lex }}$ - viable

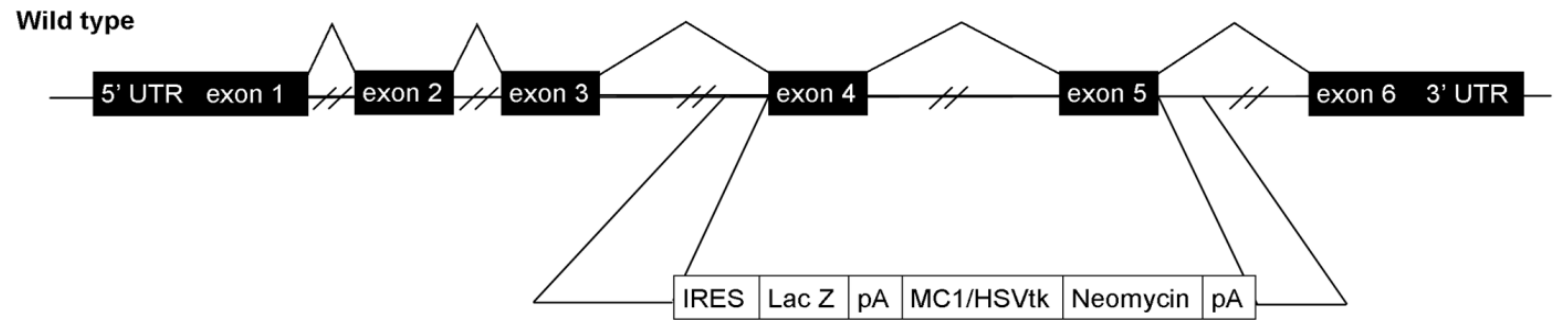

Pdgfc $^{\text {tm1Lex }}$

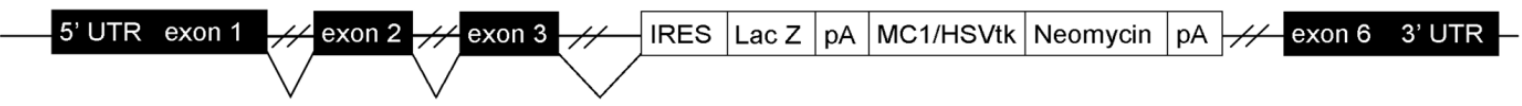

Figure 1: Design of targeting constructs to inactivate PDGF-C. a) A linear diagram of the PDGF-C protein showing the CUB (orange) and GFD (green) above the exons that correspond to these protein domains. b) The targeting strategy for Pdgft $c^{\text {tmllex }}$ mice, which removes exons 4 and 5. c) The targeting strategy for the Pdgf $f^{\text {minagy }}$ strain, which removes exon 2 Ding, et al. [5] Abbreviations : splice acceptor (SA), internal ribosomal entry site (IRES), complement components $\underline{\mathrm{C}} 1 \mathrm{r} / \mathrm{C} 1 \mathrm{~s}$ sea urchin EGF bone morphogenic protein 1 (CUB), beta galactosidase neomycin fusion protein ( $\beta$ GE0), fusion polyprotein (A) tail (pA), Lac Z gene encoding beta galactosidase (Lac Z), enhancer, promoter, and neomycin phosphotransferase (MC1/ HSVtk and Neomycin).

were further backcrossed to a C57BL/6 background six times to generate the Mendelian ratio for C57BL/6 (Table 1). These mice can be obtained from the Mutant Mouse Regional Resource Centers (http://www.mmrrc.org/index.php) supported by the NIH. Hemizygous Pdgfr $\alpha$ mice, Pdgfra ${ }^{\text {tm11(egf } p) s o r}$, were purchased from The Jackson Laboratory (stock \# 007669). Animals were housed at the University of Washington, which is an Association for the Assessment and Accreditation of Laboratory Animal Care approved facility, and all experiments were performed with the University of Washington Institutional Animal Care and Use Committee approval.

\section{Necropsy and Histology}

Homozygote Pdgfc $c^{\text {tmllex }}$ mice $(\mathrm{n}=4)$ and WT $\mathrm{C} 57 \mathrm{BL} / 6$ 
Table 1: Heterozygous $P d g f c^{\text {tmllex }}$ breeding pairs in two backgrounds produce offspring with normal Mendelian distribution.

\begin{tabular}{|c|c|c|c|c|c|}
\hline Cohort & $\begin{array}{l}\text { No. of } \\
\text { animals }\end{array}$ & $+/+$ & $P d g f c^{t m 1 l e x /+}$ & $P d g f c^{t m 1 l e x} / P d g f c^{t m 1 l e x}$ & Chi sq value \\
\hline \multicolumn{6}{|c|}{ 129Sv/EvBrd } \\
\hline observed & 99 & 26 & 50 & 23 & 0.19 \\
\hline expected & & 25 & 50 & 25 & \\
\hline \multicolumn{6}{|l|}{ C57BL/6 } \\
\hline observed & 265 & 55 & 147 & 63 & 3.7 \\
\hline \multicolumn{6}{|l|}{ male } \\
\hline observed & 146 & 29 & 83 & 34 & 3.1 \\
\hline expected & & 37 & 73 & 37 & \\
\hline \multicolumn{6}{|l|}{ female } \\
\hline observed & 119 & 26 & 64 & 29 & 0.83 \\
\hline expected & & 30 & 59 & 30 & \\
\hline
\end{tabular}

Male and female heterozygous $P d g f c^{\text {tmllex }} /+$ mice were bred and the genotypes of the resulting offspring were determined from DNA extracted from tail snips by PCR. Two different genetic backgrounds, 129SvBrd and C57BL/6, were analyzed. The expected number of pups with a specific genotype was calculated based on the total number of pups analyzed. Analysis of normal Mendelian distribution was determined by Chi square analysis with two degrees of freedom. A Chi square number greater than 5.99 is statistically significant.

littermates $(n=4)$ aged 5 and 8 weeks were euthanized by $\mathrm{CO}_{2}$ asphyxiation followed by complete necropsy. Blood was collected for chemistry and complete blood counts performed by Phoenix Central Laboratories (Everett, WA). Tissues were collected for histological analysis with immersion fixation in $10 \%$ phosphate-buffered formalin, 4-6 $\mu \mathrm{m}$ sections were made and stained with hematoxylin and eosin. Tissues examined included: lungs, esophagus, trachea, liver, kidneys, heart and great vessels, adrenal glands, gallbladder, brown and white adipose tissue, exocrine and endocrine pancreas, spleen, thyroid, submandibular, parotid and sublingual salivary glands, mesenteric lymph nodes, mesentery, preputial or clitoral glands, skeletal muscle, bladder, male accessory sex glands, testes or ovaries, haired skin, large and small intestine, glandular and non-glandular stomach, uterus and cross section of the head (eyes, middle and internal ears, oral and nasal cavities, cerebrum, cerebellum, olfactory lobes, brain stem, pituitary, tongue and teeth). All tissues were examined by a board-certified Veterinary Pathologist (PMT) and given a morphological diagnosis where applicable. Skeletons were processed by eviscerating, removing the brain and as much muscle tissue and fat as possible, followed by immersion in 95\% ethanol for at least 72 hours, placed in acetone overnight, and stained the next night at $37^{\circ} \mathrm{C}$ with a solution of $70 \%$ ethanol, $5 \%$ glacial acetic acid, $11.6 \mu \mathrm{M}$ Alcian blue, and $14.6 \mu \mathrm{M}$ Alizarin red. The following day the skeletons were washed in $95 \%$ ethanol and cleared in $2 \% \mathrm{KOH}$ for 2 days and transferred to $1 \% \mathrm{KOH}$ until fully cleared, with the solution refreshed every 2 to 3 days until soft tissues were cleared, at which point the skeletons were transferred to $100 \%$ glycerol.

\section{Genotyping}

Genomic DNA was extracted from mouse tails by incubation overnight at $55^{\circ} \mathrm{C}$ in $20 \mu \mathrm{g} / \mathrm{ml}$ Proteinase $\mathrm{K}$ (life technologies) in lysis buffer (200 mMNaCl,1\%W/VSDS, 10mMTris-HCl,1 mMEDTA $\mathrm{pH}$ 8.0). Phenol chloroform extraction was performed, followed by ethanol precipitation and resuspension in Tris-HCl 10mM EDTA $1 \mathrm{mM}$ pH 7.4 (TE). Polymerase chain reaction was carried out with $0.5 \mathrm{U}$ GemTaq (MGQuest), supplied $5 \mathrm{x}$ buffer, and dNTPs to a final concentration of $0.2 \mathrm{mM}$ with primers 5' CCTGGTCAAGCGCTGTGG 3'(200nM), 5' TCTGGATTCATCGACTGTGG 3'(200nM), 5' ACGGCTAACATGGAGCACG 3' (100 nM). All primers were designed using Oligo Calc [21]. Cycling conditions were $95^{\circ} \mathrm{C}$ for $3 \mathrm{~min}$, and $35 \mathrm{cycles}$ of $95^{\circ} \mathrm{C}$ for $20 \mathrm{sec}, 60^{\circ} \mathrm{C}$ for $30 \mathrm{sec}$, and $72^{\circ} \mathrm{C}$ for $30 \mathrm{sec}$ with a final extension at $72^{\circ} \mathrm{C}$ for $10 \mathrm{~min}$.

\section{Southern blotting}

The targeting construct was verified using standard methods as described [22]. Briefly, genomic DNA was extracted as described above and purified DNA was restriction digested using EcoR1 HF (New England Biolabs) at $37^{\circ} \mathrm{C}$ overnight. $10 \mu \mathrm{g}$ of DNA from $P d g f c^{\text {tm1lex }}$ homozygous, heterozygous, and wild-Type (WT) mice was loaded onto a Tris-acetate-EDTA (TAE) gel. Following electrophoresis, the gel was denatured in $0.4 \mathrm{M} \mathrm{NaOH}, 1.5 \mathrm{M} \mathrm{NaCl}$ for $10 \mathrm{~min}$ followed by neutralization in Tris- $\mathrm{HCl}$ with $1.5 \mathrm{M} \mathrm{NaCl}$, pH 7.4. DNA was transferred to Hybond N+ (GE Healthcare) nylon membrane in 10x SSC and cross-linked in a Stratalinker 1800 (Stratagene). Exonic DNA including exon 6 and the 3' UTR was used as a probe, amplified by 5' CCTTTTAGGTCCTTCAGTTGAGACC 3 ' and 5 ATCTATGCAAACAGGTTGGAGAAATCC 3'. The probe was labeled with $50 \mu \mathrm{Ci}\left[\alpha-{ }^{32} \mathrm{P}\right]$ dCTP using random decamers (DECAprime II, Ambion) to a specific activity $>108 \mathrm{dpm} / \mu \mathrm{g}$. The blot was prehybridized with Quickhyb (Stratagene) at $68^{\circ} \mathrm{C}$ for $30 \mathrm{~min}$ and then incubated with the denatured probe (106 dpm/ $\mathrm{ml}$ ) at $68^{\circ} \mathrm{C}$ for $2 \mathrm{hr}$. Labeled membranes were then washed in $2 x$ SSC with $0.1 \%$ SDS and $0.2 x$ SSC with $0.1 \%$ SDS at $60^{\circ} \mathrm{C}$ for 30 min each. The sizes of the digested genomic DNA were confirmed by comparison with 32P- dCTP end-labeled $\lambda$ Hin III DNA marker (Fermentas). 


\section{RT-PCR analysis}

Tissues were collected and flash frozen immediately in liquid nitrogen. RNA was extracted with Trizol (Invitrogen) as per the manufacturer's instructions, and cDNA synthesized from $0.5 \mu \mathrm{gRNA}$. Primers (F1) 5' CTCACGTGTGCTGCTACGAAGG 3' and (R3) 5' CCCTGCGATTCTCTGCTGCC 3' were used with the following conditions; $95^{\circ} \mathrm{C}$ for $3 \mathrm{~min}$, and 35 cycles of $95^{\circ} \mathrm{C}$ for 15 $\mathrm{sec}, 58^{\circ} \mathrm{C}$ for $15 \mathrm{sec}$, and $72^{\circ} \mathrm{C}$ for $30 \mathrm{sec}$ with a final extension at $72^{\circ} \mathrm{C}$ for $10 \mathrm{~min}$.

\section{Cloning}

WT and Pdgfc ${ }^{\text {tm1lex }}$ CDNA was amplified with primers 5' GCCCTCGCCCCAGTCAGC $3^{\prime}$ and 5' CTCACGTGTGCTGCTACGAAGG 3' to generate Pdgf- $c$ and $P d g f c^{\text {tm } 1 l e x}$ sequences. WT cDNA was amplified with primers 5' ATGGTGGTGAATCTAAATCTCCTC 3' and 5' CTCACGTGTGCTGCTACGAAGG 3' to generate the GFD construct. Additionally, a sequence containing a Kozak sequence and the Pdgf-c signal peptide for secretion was added to the 5' end of the GFD by Amplification (i.e., 5'GCAGAATTGCCACCATGCTCCTCCTCGGCCT-CCTCCTGCTGACATCTGCCCTGGCCGGCCAAAGAACGGGGACTCGGGCTGAGTCC3').

The Pdgf-c, PdgfC $C^{\text {tmllex}}$, and GFD sequences were cloned separately into pEF1/myc-His B plasmids (Life Technologies), but retained the termination codons which prevent translation of the myc-His tag. The plasmids were linearized with Mlu I (New England Biolabs).

\section{Transfection and production of conditioned media (CM)}

Cells [HEK293] ATCC $^{\circledR}$ CRL1573 $^{\text {mo }}$ ) were maintained at $37^{\circ} \mathrm{C}$ 95\% humidity with $5 \% \mathrm{CO}_{2}$. Transfections were performed using the calcium phosphate method as described [23], and the cells were subjected to $500 \mu \mathrm{g} / \mathrm{ml} \mathrm{G} 418$ (Life Technologies) for two weeks to select for cells expressing the following constructs: empty vector (pEF1empty), $p E F 1 P d g f^{G F D}, p E F 1 P d g f c^{m u t}$, or $p E F 1 P d g f c^{F L}$ to produce untagged proteins, $P d g f-c^{G F D}, P d g f-c^{m u t}$ and $P d g f-c^{F L}$ respectively. Confluent cultures were allowed to condition media for 24 hours, at which point media were collected and frozen at $-80^{\circ} \mathrm{C}$. Baby Hamster Kidney (BHK) cells, which do not express Pdgfra (BHK 570), and BHK cells transfected with Pdgfra (BHK R $\alpha$ ) were a kind gift from Dr. Daniel Bowen-Pope [24].

\section{Immunoblot analysis}

SDS-PAGE and immunoblotting were carried out as described [25]. For PDGF-C detection membranes were incubated with goat anti-mouse PDGF-C antibody (1:1000, AF1447 R\&D Systems) followed by incubation with an anti-goat Horseradish Peroxidase (HRP) conjugated secondary antibody and detection performed with ECL (Pierce). For ERK and p-ERK detection, membranes were first incubated with anti-phospho p44/42 MAPK (1:1000, 9101 Cell Signaling Technology) followed by an anti-rabbit conjugated secondary antibody. Primary antibody; secondary antibody complexes were detected with ECL Plus (GE Healthcare) and imaged with a Storm 840 phosphor imager (GE Healthcare). Immunoblots were then stripped with $2 \%$ SDS, $62.5 \mathrm{mM}$ Tris,
pH 6.8 with $7 \mu \mathrm{l}$ beta-mercaptoethanol per ml. Blots were then reprobed with anti-ERK1/2 pAb 7884 followed by secondary and ECL as above. Densitometry analyzes were performed using Image J software (NIH) and is presented as the relative value of the phosphorylated protein normalized to the total levels of the same protein after re-probing.

\section{Results}

\section{Homologous recombination targeting strategy}

We examined a Pdgf-c mutant mouse, $P d g f c^{\text {tm1lex }}$, in which exons 4 and 5 of the GFD are deleted using the targeting strategy depicted in Figure 1b. Surprisingly, a viable mouse resulted from this approach; in contrast to the Pdgf-c KO mice, which results in perinatal lethality $\left(P d g f c^{t m 1 n a g y}\right.$, Figure 1c). Figure 1 shows the protein domain structure of PDGF-C and compares the different targeting approaches used to generate the two mutant strains. Pdgfc ${ }^{\text {tm1lex }}$ mice were derived from a 129S5/SvEvBrd embryonic stem cell line (Lex1) targeted by homologous recombination to replace exons 4 and 5. This strategy should eliminate transcription of the entire GFD (Figure 1b). Pdgft ${ }^{\text {tm } 1 \text { nagy }}$ mice were derived from R1 embryonic stem cells targeted by homologous recombination to replace exon 2 of PDGFC with an SA-IRES- $\beta$-geo-pA cassette [5], preventing transcription downstream of $\beta$-geo, including the CUB and GFD.

\section{Verification of recombination}

To confirm that our selection cassette was targeted correctly, we performed Southern blotting on genomic DNA isolated from homozygous Pdgft $c^{\text {tmlex }}$, heterozygous Pdgfc $c^{\text {tmllex }}$, and WT littermates. Digestion of genomic DNA with EcoRI should produce a $12.4 \mathrm{~kb}$ band including exons $4,5,6$, and the 3 ' untranslated region (UTR) from WT DNA, and a $6.5 \mathrm{~kb}$ band including exon 6 and the 3' UTR in correctly targeted Pdgf $c^{\text {tm }}{ }^{\text {lex }}$ mice (Figure 2a). A ${ }^{32} \mathrm{P}$ labeled DNA probe with a complementary sequence to exon 6 and the 3' UTR of Pdgf- $c$ was used to detect these 12.4 and 6.5 $\mathrm{kb}$ bands by hybridization and bands of the expected size were detected for homozygous Pdgfc ${ }^{\text {tm1lex }}$, heterozygous Pdgfc ${ }^{\text {tm1lex }}$, and WT mice (Figure 2b).

\section{Pdgf $^{\text {tm1lex }}$ transcription and translation}

3.4 Given our Southern blotting results, we next verified production of the expected Pdgfc ${ }^{\text {tmilex }}$ transcript, which should result from transcription of exons 1, 2, and 3 of Pdgf-c (Figure 1c). Attempts to amplify a transcript containing exon 3 and the internal ribosomal entry site (IRES) (Figure 3a, primers F1 and R1) or lacZ (Figure 3a, F1 and R2) did not produce a product (data not shown), suggesting the targeting construct may have facilitated skipping the KO cassette. RT-PCR of Pdgfc ${ }^{\text {tm } 1 \text { lex }}$ cDNA using primers F1 and R3 (Figure 3a) generated a 296 bp band, consistent with a transcript in which exons 1, 2, 3 and 6 remain (Figure 3b). Sequencing this amplicon revealed that indeed the region corresponding to WT exons 4 and 5 was removed, but exon 6 was spliced in frame to the 3 ' end of exon 3 to create a truncated transcript (Figure 4). To detect the full-length coding region of 


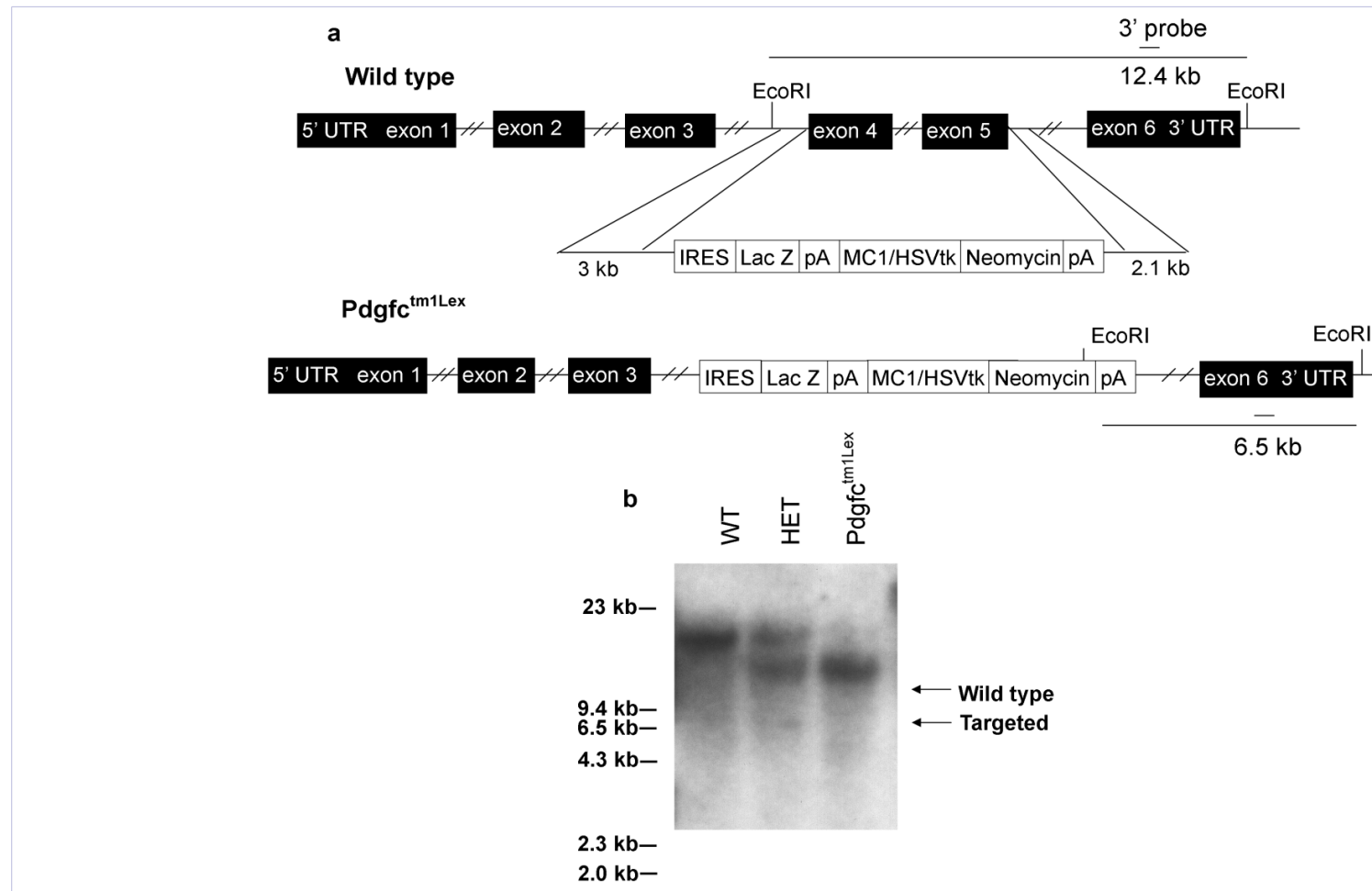

Figure 2: Recombination at the Pdgf-c locus in the Pdgfc ${ }^{\text {tm1lex }}$ mouse. a) The anticipated results from EcoR1 digestion of genomic DNA from a wild type mouse and a $P d g f c^{\text {tmllex }}$ mouse. d) Southern blot of genomic DNA digested with EcoRI from wild type, heterozygous, and homozygous $P d g f c^{t m l l e x}$ mice.
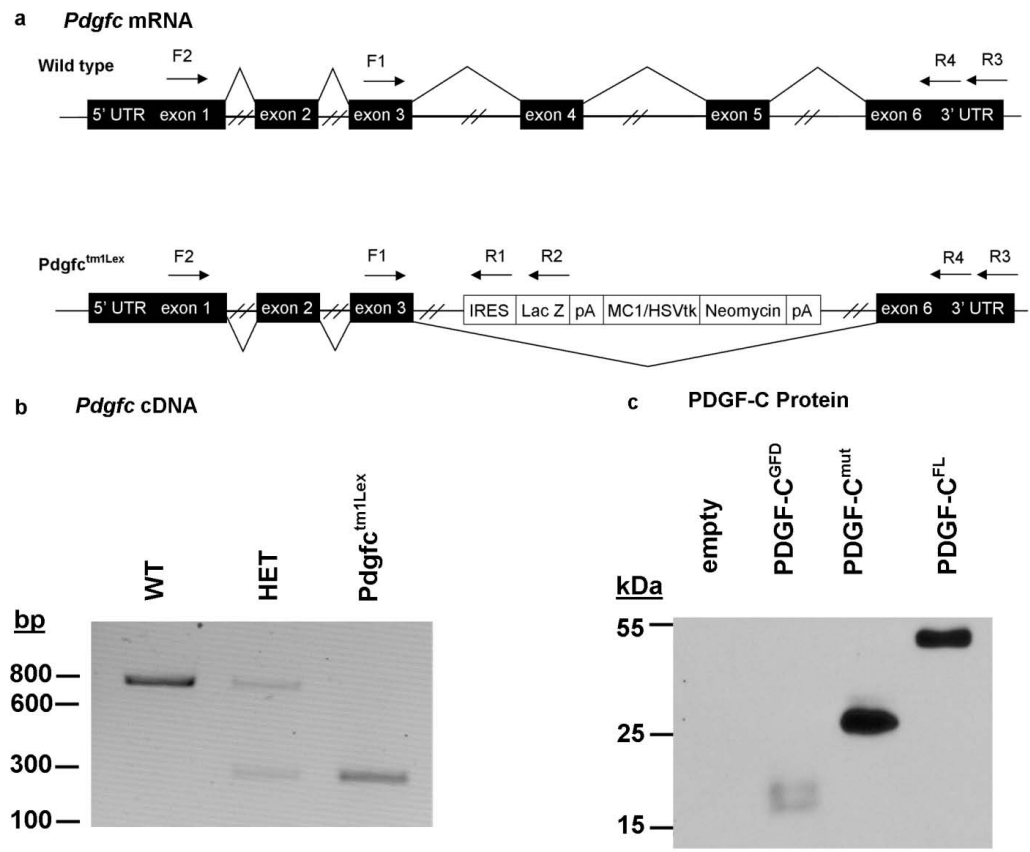

Figure 3: An alternative mRNA is transcribed from the Pdgf-c locus in Pdgfc $c^{t m 1 l e x}$ mice, and protein is expressed from this sequence. a) Diagram of the splicing of wild type and mutant PDGFC with various PCR primers (F1, F2, R1, R2, R3, and R4) used to detect transcripts. b) Amplicons generated by RT-PCR (F1 and R3) of RNA from kidneys of wild type, heterozygous, and homozygous Pdgfc $c^{\text {tmllex }}$ mice are shown, producing products of 722 bp for $P d g f-c$ and 296 bp for Pdgfctmlex. c) Immunoblot of conditioned media from transfected HEK293 cells expressing the coding sequences for an empty vector (empty), the GFD (Pdgf-c $\left.c^{G F D}\right), P d g f c^{\text {tmllex }}\left(P d g f-c^{m u t}\right)$, or full-length Pdgf-c (Pdgf- $\left.c^{F L}\right)$. The size of DNA ladder (b) and protein standards (c) are shown to the left of each image. 
PDGFC, we designed primers to anneal to the 5' and 3' UTRs, and found that the $P d g f c^{\text {tm1lex }}$ transcript retains the endogenous 5' and 3' UTRs (Figure 3a, F2 and R4) and associated regulatory elements (data not shown).

The Pdgfc ${ }^{\text {tm llex }}$ mutant transcript is expected to have an expression pattern similar to WT Pdgf-c, given that the selection cassette did not alter the $P d g f-c 5^{\prime}$ and 3 ' regulatory elements. The translated protein product of Pdgfc ${ }^{\text {tm1lex }}$ is expected to contain the C-terminal 38 amino acids of the GFD and the entire CUB domain, resulting in a predicted molecular weight of $22.5 \mathrm{kDa}$. We found that HEK293 cells transfected with plasmids expressing the coding regions for full length $P d g f-c\left(P d g f-C^{f}\right), P d g f C^{\text {tm } 1 l e x}(P d g f-$ $c^{m u t}$ ), or Pdgf-c GFD (Pdgf-C $C^{G F D}$ ) secrete proteins of the predicted molecular weights into culture media, as determined by immunoblot analysis (Figure 3c). Thus Pdgf- $c^{F L}, P d g f-C^{m u t}$ and Pdgf$c^{G F D}$, are all stable protein products.

\section{Signal transduction in response to conditioned medium}

To demonstrate that the CUB domain of Pdgf-c could an activates Pdgfr $\alpha$, we assessed ERK phosphorylation in BHK cells treated with various Conditioned Media (CM). Media were conditioned by HEK293 cells transfected with plasmids expressing Pdgf-c CUB, Pdgf-c GFD, or an empty vector control. When CM was added to BHK 570 cells, which have no Pdgfr $\alpha$, no phosphorylation of ERK was detected (data not shown). When conditioned medium $\alpha$ was cells added to BHK R $\alpha$, which have been transfected with Pdgfra, phosphorylation of ERK was detected in a time-dependent manner, with maximal phosphorylation attained after five minutes of exposure (Figure 5a). Media conditioned by HEK293 cells transfected with an empty vector control interestingly did show ERK phosphorylation in BHK R $\alpha$ cells but not in BHK 570 cells, albeit to a lesser extent than the PDGF-C constructs, suggesting that

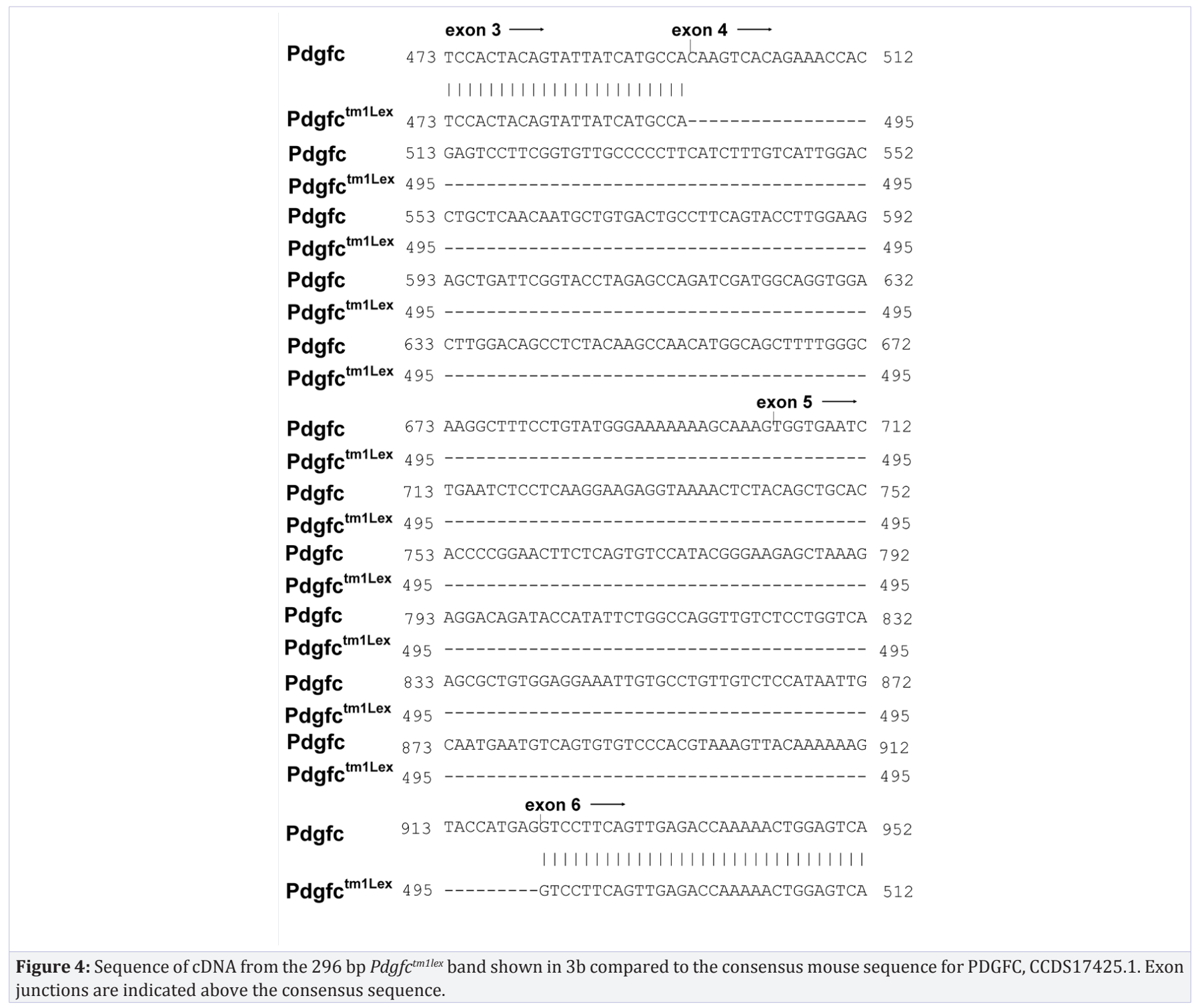

Citation: Hayes BJ, Riehle KJ, Gilbertson DG, Curtis WR, Kelly EJ, et al. (2015) The PDGFC CUB Domain Enhances Survival in PDGFC Page 6 of 11 Mutant Mice. SOJ Immunol 3(3): 1-11. DOI: http://dx.doi.org/10.15226/soji/3/3/00133 
transfection of HEK293 cells induces the release of a molecule that can signal through Pdgfr $\alpha$ When BHK R $\alpha$ cells are exposed to increasing ratio of $\mathrm{CM}$ to fresh medium we see an increase in intensity of ERK phosphorylation just as increasing the amount of recombinant $P d g f-c$ increases ERK phosphorylation (Figure $5 b$ ). CM from Pdgf-c GFD and Pdgf-c CUB domain transfected HEK 293 cells maintains some ability to induce ERK phosphorylation even at low $\mathrm{CM}$ ratio to fresh medium.

\section{Anatomic characterization}

Originally derived on a $129 \mathrm{~S} 5 /$ SvEvBrd background, $P d g f c^{\text {tmllex }}$ mice were backcrossed to a C57BL/ 6 background. Homozygous F1 C57BL/6 x 129S5/SvEvBrd Pdgfc $C^{\text {tm1lex }}$ progeny had a hunched appearance, and gross skeletal analyzes demonstrated spina bifida (Figure 5a and 5b), which was confirmed by histology (data not shown). All other organs were grossly normal. After six backcrosses, no overt anatomical differences were noted between homozygous Pdgfc ${ }^{\text {tmllex }}$ mice and WT C57BL/6 littermates, i.e. the spina bifida phenotype was rescued. Comprehensive histological analyses (see methods) conducted on 5 and 8-week old Pdgfc ${ }^{\text {tmllex }}$ homozygous mice were unremarkable or showed incidental lesions, such as dermatitis or mild multifocal extramedullary hematopoiesis in the liver, which are known to be associated with the C57BL/6 background [26]. Brains of Pdgfc ${ }^{\text {tm1lex }}$ mice were examined histologically and did not differ from those of WT littermates; clinical chemistry and blood counts were also unremarkable (data not shown).

\section{Genotype ratios}

We observed normal Mendelian ratios in the offspring of heterozygote $P d g f c^{\text {tmllex }}$ breeding pairs on two genetic backgrounds (Table 1). We did not observe premature death in Pdgfc ${ }^{\text {tm } 1 l e x}$ mice up to 12 months of age and did not see sexdependent differences in viability on the C57BL/6 background, as has been reported for Pdgfc ${ }^{\text {tminagy }}$ mice [20]. Similar to Fredriksson L, et al. [6] we observed a statistically significant decrease in body mass in C57BL/ 6 Pdgfc ${ }^{\text {tmllex }}$ mice at 3 and 7 months (a 17 to $24 \%$ decrease, data not shown). To determine whether Pdgfra necessary for normal development in Pdgf tmllex $^{\text {tm }}$ mice, we intercrossed hemizygous Pdgfr $\alpha$ mice with heterozygous $P d g f c^{t m 1 l e x}$ mice. Progeny resulting from this cross did not include mice that were homozygous Pdgf ${ }^{\text {tm1lex; }}$, hemizygous Pdgfr $\alpha$ (Table 2). Neonatal mice homozygous for Pdgf $c^{\text {tm1lex }}$ and hemizygous for Pdgfr $\alpha$ were born but failed to thrive and $100 \%$ died prior to the age of weaning. This result is surprising, as hemizygous Pdgfra mice are phenotypically normal $[1,27]$; we thus conclude that a

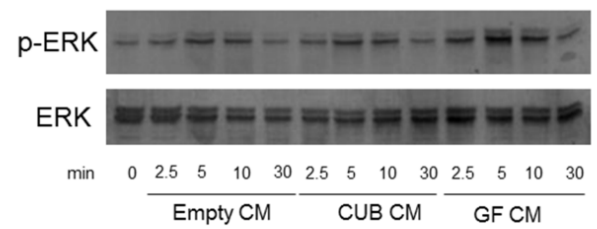

b

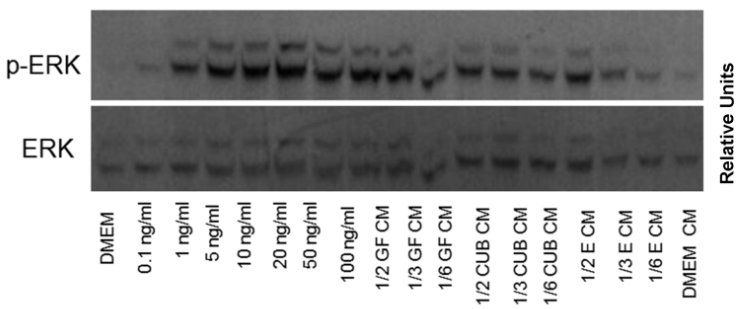

Densitometry

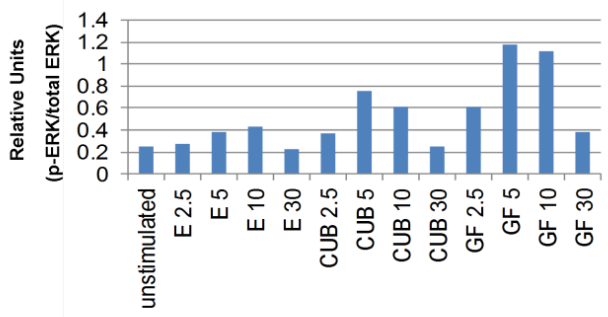

Densitometry

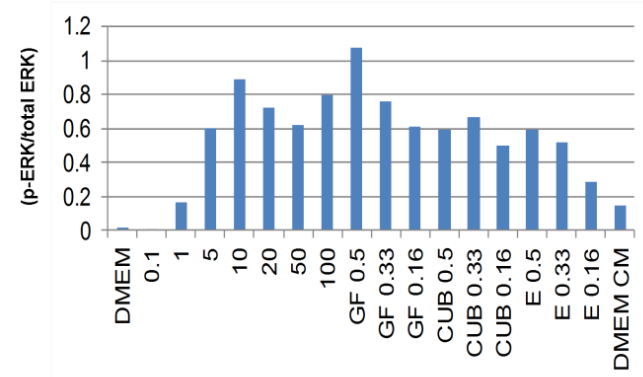

Figure 5: PDGF-C CUB domain stimulates modest ERK phosphorylation in BHK cells expressing PDGFR $\alpha$. HEK293 cells were transfected with an empty vector (empty), the CUB domain of PDGF-C (CUB), or the growth factor domain of PDGF-C (GF) and the conditioned media (CM) was transferred to BHK cell lines expressing PDGFR $\alpha$.

a) Time-dependent ERK phosphorylation by PDGF-C CUB, PDGF-C GF, or empty vector (E). Immunoblot phospho-ERK1/2 in BHK R $\alpha$ cells transfected with Pdgfra after addition of CM. corresponding densitometry measurements are shown to the right.

b) Varying amounts of GF CM, CUB CM, and empty vector CM with non-CM stimulated ERK phosphorylation while DMEM (left lane) and DMEM conditioned from non-transfected HEK293 cells weakly phosphorylated ERK. Corresponding densitometry measurements are shown to the right. Note: no phosphorylation of ERK was seen in the parental BHK cells under similar conditions (data not shown). 
Table 2: Heterozygous $p d g f c^{\text {tmllex }}$ breeding pairs with one copy of $p d g f r \alpha$ do not produce $p d g f c^{\text {tmllex }}$ homozygous and hemizygous $p d g f r \alpha$ offspring.

\begin{tabular}{|l|l|l|l|l|l|l|l|l|}
\hline Cohort & No. of animals & $\begin{array}{l}\text { Pdgfc+/+ } \\
\text { Pdgfr } \alpha+/-\end{array}$ & $\begin{array}{l}\text { Pdgfc+/- } \\
\text { Pdgfr } \alpha+/-\end{array}$ & $\begin{array}{l}\text { Pdgfc-/- } \\
\text { Pdgfr } \alpha+/-\end{array}$ & $\begin{array}{l}\text { Pdgfc }+/+ \\
\text { Pdgfr } \alpha+/+\end{array}$ & $\begin{array}{l}\text { Pdgfc+/- } \\
\text { Pdgfr } \alpha+/+\end{array}$ & $\begin{array}{l}\text { Pdgfc-/- } \\
\text { Pdgfr } \alpha+/+\end{array}$ & Chi sq value \\
\hline observed & 127 & 14 & 27 & 0 & 20 & 50 & 16 \\
\hline expected & & 16 & 32 & 16 & 16 & 32 & 16 \\
\hline
\end{tabular}

Male and female heterozygous $P d g f r^{t m 1 l e x} /+$ mice, with a single copy of $P d g f r \alpha$, were bred, and the genotypes of the resulting offspring were determined from DNA extracted from tail snips by PCR. The expected number of pups with a specific genotype was calculated based on the total number of pups analyzed. Analysis of normal Mendelian distribution was determined by Chi squared analysis with five degrees of freedom. A Chi square number greater than 11.07 is statistically significant. $P d g f c-/$ - represents $P d g f^{\text {tm } 1 l e x} /{ }^{\text {tm1lex }}$ mice.

a

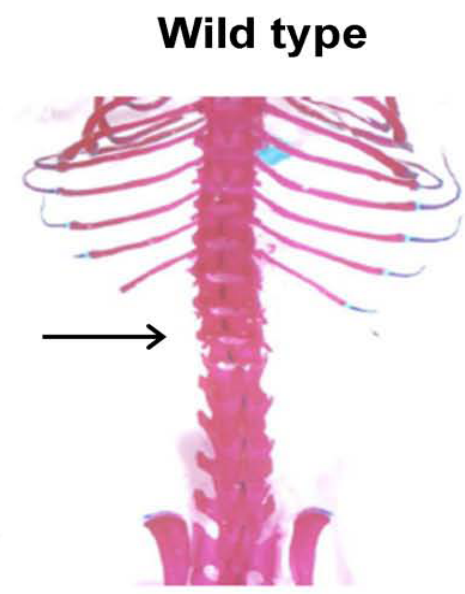

b

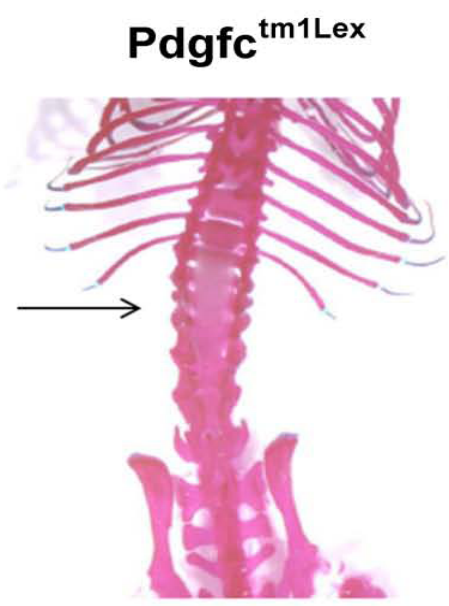

Figure 6: Anatomic characterization of $P d g f^{t m \text { llex }}$ mice.

a) 129Sv/EvBrd x C57BL/6 F1 wild type mice have normal thoracic and lumbar vertebrae.

b) Spina bifida in the thoracic and lumbar region (arrow) of $P d g f c^{t m 1 l e x}$ homozygous mice.

$P d g f c^{t m 1 l e x}$ viability requires two alleles of Pdgfr $\alpha$. Histological and gross anatomical analysis of these neonatal animals revealed severe spina bifida encompassing a region from vertebrae T11 to L1 (Figure 6 a-c). Microscopic analysis revealed demyelinated spinal cords with excessive blood infiltration. Like Pdgfc ${ }^{\text {tm1nagy }}$ mice, homozygous Pdgf $c^{\text {tm1lex; }}$ hemizygous Pdgfra mice had palatoschisis. These genetic data indicate that PDGF-C ${ }^{\text {mut }}$ interacts with Pdgfra in some novel way, as Pdgfc $c^{\text {tminagy }}$ mice have reduced viability, while $P d g f^{t m 1 l e x}$ mice have lethality only in conjunction with hemizygous Pdgfra.

\section{Discussion}

PDGF signaling is essential for normal development. In this study we analyzed a new PDGF-C mutant mouse strain and demonstrate that the homologous recombination strategy in this mouse results in an alternatively spliced mutant form of Pdgf- $c$ that lacks over $60 \%$ of the RNA encoding the GFD, but expresses the entire portion of RNA encoding the CUB domain. This truncated transcript likely results from pre-mRNA splicing, which is a complicated event relying on proper sequence of both the $3^{\prime}$ and 5' ends of exons, as well as the proper sequence of the exons themselves [28]. The targeting construct in Pdgfc $c^{\text {tm1lex }}$ used the endogenous 3' splice acceptor sequence of exon 4 , but the remainder of exon 4 was replaced with viral and bacterial sequences (IRES and Lac Z, respectively), which are incompatible with eukaryotic splicing machinery. The removal of the 3' end of exon 4 makes splicing at this particular sequence less likely, due to the inability of U1 to enhance splice site recognition [29]. The 14 bases remaining of the endogenous exon 4 sequence are likely insufficient to promote splicing, and thus lead to exon skipping in $P d g f c^{\text {tm1lex }}$ mice.

The presumed Pdgf-c protein that is translated from $P d g f c^{\text {tmllex }}$ should not have any GFD function, as paralogs of PDGF-C, PDGF-A and PDGF-B, require cysteines for dimerization and intramolecular and intermolecular folding [14,15]. We hypothesized that the Pdgfc $c^{\text {tmllex }}$ protein product (Pdgf- $c^{\text {mut }}$ ), lacking 6 of the 8 conserved cysteines, would have compromised or absent GFD signal transduction activity. Pdgf-c ${ }^{m u t}$ is also missing the paralogous loops, I and II of the GFD, which are necessary for receptor binding by the PDGF-C paralog, Pdgf- $b$ $[16,18]$. Loop I has also been shown to be critical for receptor activation by the PDGF paralogs Placental Growth Factor (PIGF) and Vascular Endothelial Growth Factor B (VEGFB), as well as the viral protein VEGFE $_{\mathrm{NZ}-7}[30,31]$. Loop III remains in PDGF$\mathrm{C}^{\text {mut }}$, but is not expected to bind Pdgfr $\alpha$ in the absence of loops I, II and the cysteine required to form loop III. Additionally, PDGF- 

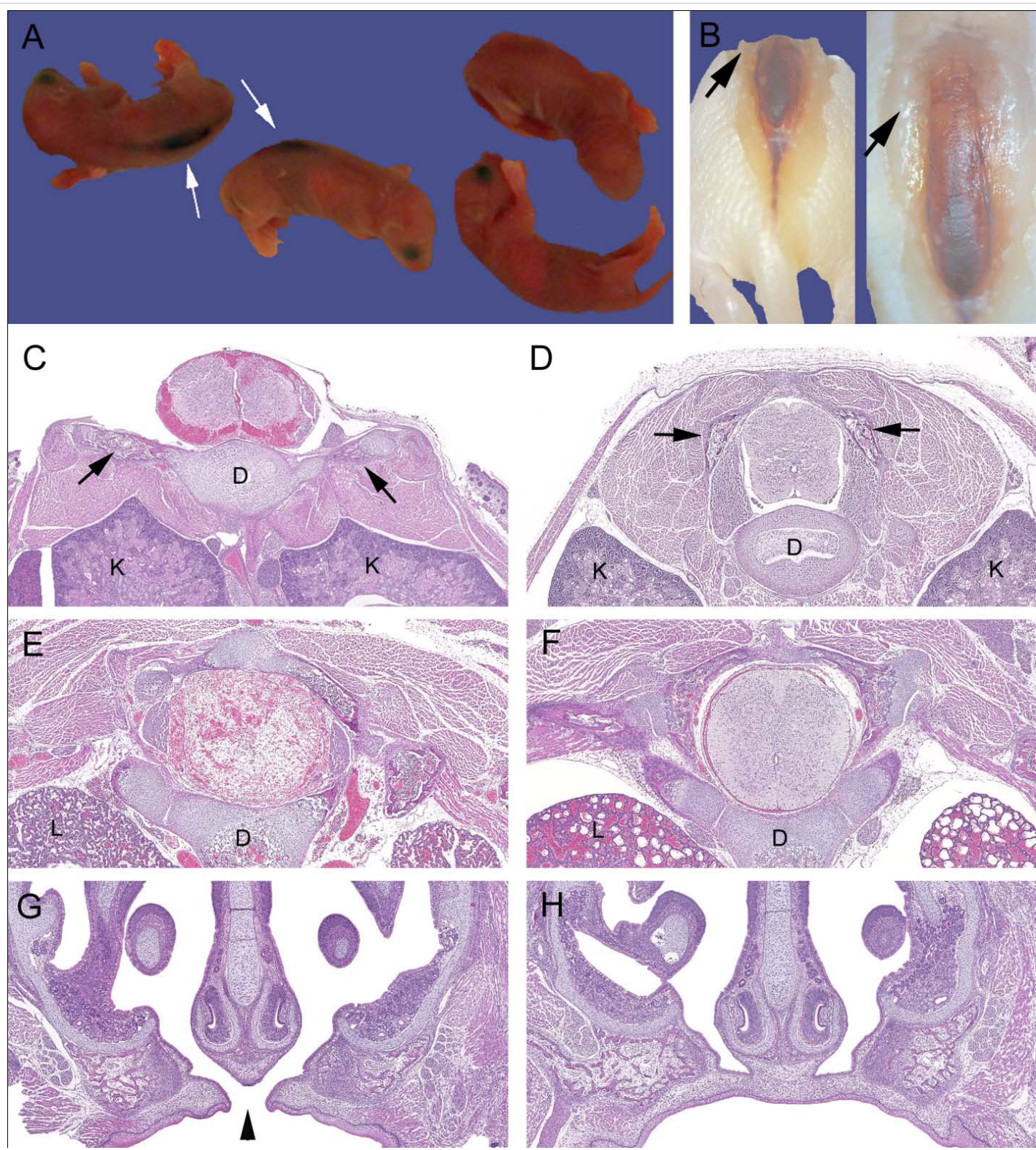

Figure 7: Developmental divergence between wild-type and homozygous Pdgf $c^{\text {tmllex; }}$, hemizygous Pdgfr $\alpha$ mice.

a) Live P0 pups. Homozygous Pdgf $c^{\text {tmllex }}$, hemizygous Pdgfra mice have dark brown linear protrusions at the level of lumbar spine (white arrows).

b) Formalin-fixed homozygous Pdgfc ${ }^{t m 1 l e x}$; hemizygous Pdgfr $\alpha$ P0 pup with dorsal skin dissected away. Note the protruding and incompletely closed vertebral lamina (arrows) and dark meningomyelocele.

c) And d) Histologic sections through the level of the lumbar spine with kidneys (K) and vertebral body or disc (D) indicated for orientation.

c) Homozygous Pdgf $c^{\text {tmllex; }}$, hemizygous PDGFR $\alpha$ pup represented in b) the spinal cord and meninges have extensive hemorrhages. The arrow indicates misaligned lamina and there are no dorsal vertebral processes, epaxial skeletal muscles, or abundant subcutaneous tissue covering the spinal cord (contrast to d).

d) Normal thoracic anatomy with proper orientation of vertebral lamina (arrows) and complete enclosure of the spine. Note the skin was removed during dissection.

e) and f) Histological sections of the thoracic spine with lungs (L) indicated for orientation. e) Homozygous Pdgff $c^{\text {tmllex; }}$ hemizygous PDGFR $\alpha$ pups have severe ascending hemorrhagic myelomalacia.

f) Corresponding cross section of a wild type mouse. Note that the pulmonary hemorrhage and extravasated red blood cells in the meninges are secondary to euthanasia.

g) and h) Cross section of the sinuses and hard palate at the level of the vomeronasal organ (T1).

g) Palatoschisis (cleft palate) is present in the Homozygous Pdgfc ${ }^{\text {tmllex; }}$ hemizygous PDGFR $\alpha$ mouse (arrowhead).

h) Wild-type mouse.

Citation: Hayes BJ, Riehle KJ, Gilbertson DG, Curtis WR, Kelly EJ, et al. (2015) The PDGFC CUB Domain Enhances Survival in PDGFC 
$\mathrm{C}^{\text {mut }}$ lacks arginine 231, which has specifically been shown to be necessary for cleavage of the CUB domain, and this cleavage has been suggested to be critical for Pdgfra activation. [9,10]. All of these structural differences suggest that PDGF-C ${ }^{\text {mut }}$ would not interact with Pdgfra in the same manner as does PDGF-C. Our data suggest CUB-mediated activation of Pdgfr $\alpha$ and raise the possibility that the CUB domain interacts with Pdgfra outside of the ligand binding pocket or perhaps interacts with the receptor in conjunction with other proteins to influence cell signaling.

CUB domains of proteins other than PDGF-C are involved in a diverse range of functions, including complement activation, developmental patterning, tissue repair, axon guidance and angiogenesis, fertilization, hemostasis, inflammation, neurotransmission, receptor-mediated endocytosis, and tumor suppression [32-34]. CUB domains have even been reported to be involved in cell signaling [35-37]. The human version of the PDGF-C CUB domain stimulates proliferation of human coronary artery smooth muscle cells [38]. Our results show that the PDGF-C CUB domain simulates modest ERK phosphorylation in BHK cells that express Pdgfr $\alpha$, but not in the parental BHK 570 cells, which do not express the receptor. These results suggest that the CUB domain interacts with Pdgfr $\alpha$ to transduce intracellular signaling events. This notion is supported by the observations that pups who are homozygous Pdgfc ${ }^{\text {tm1lex; }}$, hemizygous Pdgfr $\alpha$ (Table 2) were not viable. The precise mechanisms involved with CUB domainreceptor interactions and subsequent signal transduction events are unknown. Relative to the PDGF-C GFD, however, the CUB domain seems to be less potent in activating the receptor. In vivo, it is possible that in the absence of the GFD, the CUB domain transduces sufficient $P d g f r \alpha$ activity to prevent perinatal lethality in homozygous Pdgfc ${ }^{\text {tm1lex }}$ mice.

Genetic background appears to have a profound effect on the PDGF signaling pathway in mice. For example, the Patch mutant mouse $(P h)$, in which a segment of chromosome 5 including Pdgfra is deleted, has a phenotype that varies with background, as C57BL/6 homozygous $P h$ mice die earlier in development than do Ph mice on a CBA, or BALB/C background [1]. Likewise, the severity of abnormalities in targeted Pdgfra null mice varies depending on background, with only DBA mice surviving until birth $[1,39]$. Though our observations may be due in part to strain differences, Pdgfc ${ }^{\text {tm1lex }}$ and Pdgfc $c^{\text {tm1nagy }}$ mice appear to have different viabilities and phenotypes. Pdgfcc $C^{\text {tm1nagy }}$ mice have developmental abnormalities on a 129S1/Sv*129X/ SvJ background, specifically a lethal palate formation defect [5]. These mice are viable on a C57BL/ 6 background but have brain abnormalities $[19,20]$. Conversely, Pdgfcc ${ }^{\text {tm1lex }}$ mice are viable in two backgrounds, C57BL/6 x 129S5/SvEvBrd and C57BL/6 though two alleles of $P d g f c r \alpha$ are necessary for their viability. Mice that are homozygous for Pdgfcc ${ }^{\text {tm1lex }}$ and hemizygous for Pdgfcra die within days of birth and have severe spina bifida. Our results suggest that the CUB domain of Pdgfc-c performs a function in Pdgfcr $\alpha$ signaling. Future experiments are needed to determine whether PdgfcC $C^{\text {tm1nagy }}$ mice on C57BL/ 6 background require two $P d g f c r \alpha$ alleles. The availability of $P d g f c c^{\text {tm } 1 l e x}$ mice will facilitate additional genetic screens, either alone or in conjunction with
Pdgfc ${ }^{\text {tm1nagy }}$ mice or other mutants, to determine the biological functions of the PDGF-C CUB domain.

\section{Acknowledgments}

Grant support: NHLBI, Cardiovascular Pathology training grant (NIH-HL007312) and HHMI program in Molecular Medicine (BJH), Herbert Coe Foundation, American Surgical Association Foundation, and the American College of Surgeons (KJR), Drug Metabolism Transport and Pharmacogenetics Research Fund in the School of Pharmacy, University of Washington (EJK), NCI, mechanisms of PDGF-C induced HCC (NIH-CA127228) (JSC).

\section{Declarations}

All animal studies described in this manuscript were performed with University of Washington Institutional Animal Care and Use Committee approval. The University of Washington is an Association for the Assessment and Accreditation of Laboratory Animal Care approved facility.

\section{Sources of Support}

This work was supported by the NHLBI, Cardiovascular Pathology training grant (NIH-HL007312) and HHMI program in Molecular Medicine (BJH), Herbert Coe Foundation, American Surgical Association Foundation, Fred Hutchinson CRC New Investigator Award and the American College of Surgeons (KJR), Drug Metabolism Transport and Pharmacogenetics Research Fund in the School of Pharmacy, University of Washington (EJK), NCI, Mechanisms of PDGF-C induced HCC (NIH-CA127228) (JSC).

\section{References}

1. Soriano P. The PDGF alpha receptor is required for neural crest cell development and for normal patterning of the somites. Development. 1997;124(14):2691-2700.

2. Soriano P. Abnormal kidney development and hematological disorders in PDGF beta-receptor mutant mice. Genes Dev. 1994;8(16):18881896.

3. Boström H, Willetts $\mathrm{K}$, Pekny $\mathrm{M}$, et al. PDGF-A signaling is a critical event in lung alveolar myofibroblast development and alveogenesis. Cell. 1996;85(6):863-873.

4. Levéen P, Pekny M, Gebre-Medhin S, Swolin B, Larsson E, Betsholtz C, et al. Mice deficient for PDGF B show renal, cardiovascular, and hematological abnormalities. Genes Dev. 1994;8(16):1875-1887.

5. Ding H, Wu X, Bostrom H, Kim I, Wong N, Tsoi B, et al. A specific requirement for PDGF-C in palate formation and PDGFR-alpha signaling. Nature genetics. 2004;36(10):1111-1116.

6. Fredriksson L, Li H, Eriksson U. The PDGF family: four gene products form five dimeric isoforms. Cytokine \& Growth Factor rev. 2004;15(4):197-204.

7. Tallquist MD, Soriano P. Cell autonomous requirement for PDGFR alpha in populations of cranial and cardiac neural crest cells. Development 2003;130(3):507-518.

8. Betsholtz C, Johnsson A, Heldin CH, Westermark B, Lind P, Urdea MS, et al. cDNA sequence and chromosomal localization of human plateletderived growth factor A-chain and its expression in tumour cell lines. Nature. 1986;320(6064):695-699. 
9. Li X, Pontén A, Aase K, Karlsson L, Abramsson A, Uutela M, et al. PDGF-C is a new protease-activated ligand for the PDGF alpha-receptor. Nat Cell Biol. 2000;2(5):302-9.

10. Gilbertson DG, Duff ME, West JW, Kelly JD, Sheppard PO, Hofstrand $\mathrm{PD}$, et al. Platelet-derived growth factor C (PDGF-C), a novel growth factor that binds to PDGF alpha and beta receptor. J Biol Chem. 2001;276(29):27406-27414.

11. Bergsten E, Uutela M, Li X, Pietras K, Ostman A, Heldin CH, et al PDGF-D is a specific, protease-activated ligand for the PDGF betareceptor. Nat Cell Biol. 2001;3(5):512-516.

12. LaRochelle WJ, Jeffers M, McDonald WF, Chillakuru RA, Giese NA, Lokker NA, et al. PDGF-D, a new protease-activated growth factor. Nat Cell Biol. 2001;3(5):517-521.

13. Reigstad LJ, Sande HM, Fluge $\varnothing$, Bruland O, Muga A, Varhaug JE et al. Platelet-derived growth factor (PDGF)-C, a PDGF family member with a vascular endothelial growth factor-like structure. J Biol Chem. 2003;278(19):17114-17120.

14. Sauer MK, Donoghue DJ. Identification of nonessential disulfide bonds and altered conformations in the $\mathrm{v}$-sis protein, a homolog of the $\mathrm{B}$ chain of platelet-derived growth factor. Mol Cell Biol. 1988;8(3):10111018.

15. Kenney WC, Haniu M, Herman AC, Arakawa T, Costigan VJ, Lary J, et al. Formation of mitogenically active PDGF-B dimer does not require interchain disulfide bonds. J Biol Chem. 1994;269(16):12351-9.

16. Kreysing J, Ostman A, van de Poll M, et al. Identification of three amino acid residues in the B-chain of platelet-derived growth factor with different importance for binding to PDGF alpha- and beta-receptors. FEBS Lett. 1996;385(3):181-184.

17. Fenstermaker RA, Poptic E, Bonfield TL, Knauss TC, Corsillo L, Piskurich JF, et al. A cationic region of the platelet-derived growth factor (PDGF) A-chain (Arg159-Lys160-Lys161) is required for receptor binding and mitogenic activity of the PDGF-AA homodimer. J Biol Chem. 1993;268(14):10482-10489.

18. Andersson M, Ostman A, Kreysing J, Bäckström G, Van de Poll M, Heldin $\mathrm{CH}$. Involvement of loop 2 of platelet-derived growth factorAA and -BB in receptor binding. Growth Factors 1995;12(2):159-64.

19. Fredriksson L, Nilsson I, Su EJ, Andrae J, Ding H, Betsholtz C, et al. Platelet-derived growth factor $\mathrm{C}$ deficiency in C57BL/6 mice leads to abnormal cerebral vascularization, loss of neuroependymal integrity, and ventricular abnormalities. Am J Pathol. 2012;180(3):11361144. doi: $10.1016 /$ j.ajpath.2011.12.006.

20. Eitner F, Bücher E, van Roeyen C, Kunter U, Rong S, Seikrit C, et al. PDGF-C is a proinflammatory cytokine that mediates renal interstitial fibrosis. J Am Soc Nephrol. 2008;19(2):281-289. doi: 10.1681/ ASN.2007030290.

21. Kibbe WA. OligoCalc: an online oligonucleotide properties calculator. Nucleic Acids Res. 2007;35(Web Server issue):W43-6.

22. Crouthamel MH, Kelly EJ, Ho RJ. Development and characterization of transgenic mouse models for conditional gene knockout in the bloodbrain and blood-CSF barriers. Transgenic Res. 2012;21(1):113-130. doi: $10.1007 / \mathrm{s} 11248-011-9512-\mathrm{z}$.

23. Sambrook J, Russell DW. Calcium-phosphate-mediated Transfection of Eukaryotic Cells with Plasmid DNAs. CSH Protoc 2006;2006(1). doi: 10.1101/pdb.prot3871.
24. Ferns GA, Sprugel KH, Seifert RA, Bowen-Pope DF, Kelly JD, Murray $\mathrm{M}$, et al. Relative platelet-derived growth factor receptor subunit expression determines cell migration to different dimeric forms of PDGF. Growth Factors. 1990;3(4):315-3.

25. Campbell JS, Riehle KJ, Brooling JT, Bauer RL, Mitchell C, Fausto N. Proinflammatory cytokine production in liver regeneration is Myd88dependent, but independent of Cd14, Tlr2, and Tlr4. J Immunol. 2006;176(4):2522-2528.

26. Sundberg JP, Taylor D, Lorch G, Miller J, Silva KA, Sundberg BA et al. Primary follicular dystrophy with scarring dermatitis in C57BL/6 mouse substrains resembles central centrifugal cicatricial alopecia in humans. Vet Pathol. 2011;48(2):513-524. doi: $10.1177 / 0300985810379431$

27. Hamilton TG, Klinghoffer RA, Corrin PD, Soriano P. Evolutionary divergence of platelet-derived growth factor alpha receptor signaling mechanisms. Mol Cell Biol. 2003;23(11):4013-4025.

28. Reed R, Maniatis T. A role for exon sequences and splice-site proximity in splice-site selection. Cell. 1986;46(5):681-690.

29. Yue BG, Akusjarvi G. A downstream splicing enhancer is essential for in vitro pre-mRNA splicing. FEBS Lett. 1999;451(1):10-14.

30. Anisimov A, Leppänen VM, Tvorogov D, et al. The basis for the distinct biological activities of vascular endothelial growth factor receptor-1 ligands. Sci Signal. 2013;6(282):ra52 doi: 10.1126/scisignal.2003905.

31. Kiba A, Yabana N, Shibuya M. A set of loop-1 and -3 structures in the novel vascular endothelial growth factor (VEGF) family member, VEGF-ENZ-7, is essential for the activation of VEGFR-2 ? signaling. J Biol Chem 2003;278(15):13453-13461.

32. Thielens NM, Bersch B, Hernandez JF, et al. Structure and functions of the interaction domains of $\mathrm{C} 1 \mathrm{r}$ and $\mathrm{C} 1 \mathrm{~s}$ : keystones of the architecture of the C1 complex. Immunopharmacology. 1999;42(1-3):3-13.

33. Nakamura F, Goshima Y. Structural and functional relation of neuropilins. Adv Exp Med Biol. 2002;515:55-69

34. Mahoney DJ, Mikecz K, Ali T, et al. TSG-6 regulates bone remodeling through inhibition of osteoblastogenesis and osteoclast activation. J Biol Chem. 2008;283(38):25952-25962. doi: M802138200 [pii] 10.1074/jbc.M802138200.

35. Lee HX, Mendes FA, Plouhinec JL, et al. Enzymatic regulation of pattern: BMP4 binds CUB domains of Tolloids and inhibits proteinase activity. Genes Dev. 2009;23(21):2551-2562. doi: 10.1101/gad.1839309.

36. Hollway GE, Maule J, Gautier P, et al. Scube2 mediates Hedgehog signalling in the zebrafish embryo. Dev Biol. 2006;294(1):104-118.

37.Wu YY, Peck K, Chang YL, et al. SCUBE3 is an endogenous TGF- $\beta$ receptor ligand and regulates the epithelial-mesenchymal transition in lung cancer. Oncogene. 2011;30(34):3682-3693. doi: onc201185 [pii] 10.1038/onc.2011.85.

38. Dijkmans J, Xu J, Masure S, et al. Characterization of platelet-derived growth factor-C (PDGF-C): expression in normal and tumor cells, biological activity and chromosomal localization. The international journal of biochemistry \& cell biology. 2002;34(4):414-26.

39. McKinnon RD, Waldron S, Kiel ME et al. PDGF alpha-receptor signal strength controls an RTK rheostat that integrates phosphoinositol 3'-kinase and phospholipase Cgamma pathways during oligodendrocyte maturation. J Neurosci. 2005;25(14):3499-3508. 